
\title{
$\begin{array}{ll}\text { Research Square } & \begin{array}{l}\text { Preprints are preliminary reports that have not undergone peer review. } \\ \text { They should not be considered conclusive, used to inform clinical practice, } \\ \text { or referenced by the media as validated information. }\end{array}\end{array}$
}

\section{Finite element analysis of the optimal configuration of bridging combined internal fixation system in the treatment of vancouver B1 periprosthetic femoral fractures.}

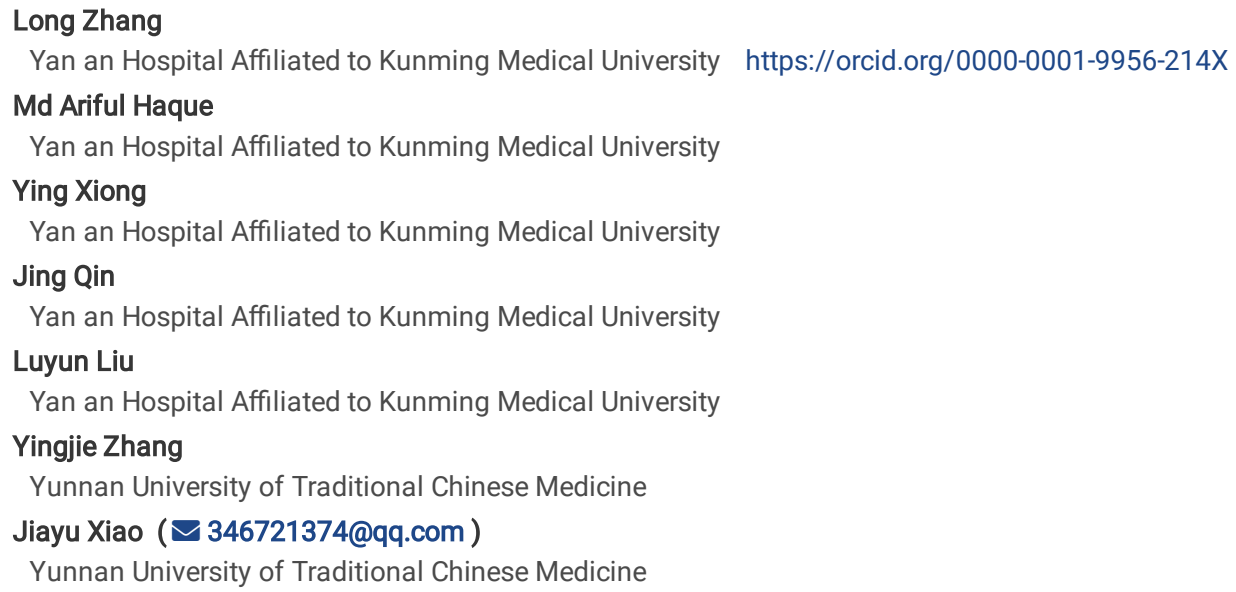




\section{Abstract}

Background and Objective: The incidence of periprosthetic fracture increases with the increase of total hip arthroplasty. The treatment of periprosthetic fracture is always a difficult point. The bridged combined internal fixation system (Ortho-bridge System, OBS) is well adapted to the characteristics of periprosthetic fractures.In this study, finite element analysis was used to evaluate the optimal configuration of OBS for fixation of Vancouver B1 periprosthetic femoral fractures.

Methods: A three-rod combination OBS fixation model was established to evaluate the optimal position of the third rod, the cross Angle of proximal screws, the diameter of the connecting rod, and the number of screws. Femoral displacement and the maximum Von Mises (equivalent) stress of OBS were used as evaluation indexes.

Results: The third rod is located at $35 \mathrm{~mm}$ below the lateral fovea of the femur and the minimum Von Mises peak stress of OBS, which is the best location for placing the third rod. It is feasible for proximal screw intersection Angle to be about $71^{\circ}$ and $84^{\circ}$. To fix the strength, the $6 \mathrm{~mm}$ connecting rod is better. Considering the number of screws scheme comprehensively, scheme $D$ is the best number of screws scheme.

Conclusion: The personalized and diversified fixation mode of OBS is well adapted to the characteristics of periprosthetic fracture and provides an effective means for the treatment of periprosthetic femoral fracture.

Statement :

I confirm that this manuscript has not been published or presented elsewhere in part or entirety and is not under consideration by another journal. However, you may notice that a preprint has been published in research square https://www.researchsquare.com/article/rs-661745/v1, "Finite element analysis of the optimal configuration of bridging combined internal fixation system in the treatment of Vancouver B1 periprosthetic femoral fractures". But it's not published or under consideration by any journal.

\section{Introduction}

As the elderly population increases, so do the incidences of hip related injuries caused by falls and osteoporosis. The field of surgical orthopedic medicine has been able to meet the increasing demands of a growing elderly population, particularly one that makes up the majority of the THA patients. The THA demand is expected to increase by $174 \%$ to 572,000 procedures by 20301 . As the number of THA patients increase, the demand for progress in the development of the best surgical technologies and practices are also set to increase. One of

the primary aims is to improve the THA patient's quality of life so that patient will not have reoccurring injuries in the area that require invasive surgical procedures and extensive recovery and rehabilitation time. Unfortunately, there has been an increase in post-THA problems, including Periprosthetic fracture (PPF)2. Abdel M P et al. reviewed 32,644 patients undergoing primary total hip arthroplasty and found that the incidence of intraoperative femoral fractures was $1.7 \%$ and that PPF was $3.5 \% 3$. A prediction model shows that the number of PPF will increase by an average of $4.6 \%$ per decade from 2015 to 2060 , with a high clinical and economic burden 5.

The treatment of PPFs is complex due to artificial Vancouver type B1 hip prostheses and the poor initial phys-ical condition of patients with PPF6. The treatment is varied due to the plethora of circumstances based on the uniqueness of the patients previous and existing conditions. An accurate classification is significant for managing PPE of the artificial hip, and the Vancouver classification is currently the most commonly used7. The Vancouver B1 fracture is a stable prosthetic fracture with no loss of bone mass and is treated with internal fixation as the pre- ferred treatment8-10. Tanvir Khan reviewed 6,131 patients with PFF and found a high risk of death, with a 5-year mortality rate of $60 \%$ in the highest risk group 11 . The plate series is the standard treatment method, which can be combined with the ring ligation, and has achieved a particular effect12-15. The advantages of using a locking compression plate in the treatment of PPF are direct exposure and reduction. The disadvantages are soft tissue dissection and the difficulty obtaining bicortical screw fixation due to intramedullary prosthesis occlusion. Lock- ing compression plate can be combined with Minimally invasive plate osteosynthesis (MIPO) to treat PPFs and maintain bone vitality 16 . Some scholars suggest additional methods such as allogeneic bone plates and fixation of at least 10 cortices 17-20. The most common complications of existing internal fixation methods included fixation failure and nonunion, with $4.4 \%$ and $3.9 \%$ rates, respectively 21 .

The OBS is a composite structure composed of the fixed block, fixed rod, and screw. The selected blocks include single side single hole, single side double hole, double rod single hole, double rod double hole, and special-shaped blocks adapted to different anatomical positions; Fixed rod includes unequal diameter; The screws include locking screws and standard screws (see Figure 1). Its design concept advocates individualization, diversification and systematization of internal orthopedic fixation, with the advantages of individualized shaping, free combination, three-dimensional fixation, elastic fixation, etc. This study analyzed the biomechanical properties of different varieties of bridging combined internal fixation systems in the treatment of PPFs by finite element analysis to determine the optimal configuration of nails, rods, and blocks.

\section{Materials And Methods Finite element model establishment}

The bone model was derived from a 48-year-old healthy male volunteer whose CT data were collected. He had no skeletal lesions, no previous surgery history, no tumor history, and no drugs affecting bone metabolism in recent years. According to the size parameters of the femoral prosthesis and the OBS, the threedimensional models of the femoral prosthesis and the OBS were established and assembled. The fracture line was simulated at the distal end of the femoral 
prosthesis stem (see Figure 2). For the material properties of cortical bone, elastic modulus $\mathrm{Ex}=\mathrm{Ey}=7.00 \mathrm{GPa}, \mathrm{Ez}=11.50 \mathrm{GPa}$, shear modulus $\mathrm{Gxy}=2.60 \mathrm{GPa}, \mathrm{Gyz}=\mathrm{Gxz}=3.50 \mathrm{GPa}$, and Poisson's ratio was 0.4 . The elastic modulus of cancellous bone was $0.40 \mathrm{GPa}$, and the Poisson's ratio was 0.3 . The femoral stem, connecting rod, and screw were made of titanium alloy with an elastic modulus of 110.00GPa and a Poisson's ratio

of 0.3. The connecting block comprises cobalt-chromium molybdenum alloy with an elastic modulus of 210.00GPa and a Poisson's ratio of 0.322 . Tetrahedral 10-node units were used to mesh the above structures. The mesh size of cortical bone was $3 \mathrm{~mm}$, the mesh size of cancellous bone and the femoral stalk was

$2 \mathrm{~mm}$, and the mesh size of connecting rods, screws, and connecting blocks was $1.5 \mathrm{~mm}$. There were 249,285 mesh units and 390,122 nodes in total. The binding contract between the screw and cortical bone, screw and joint block, joint block and rod, and femoral stem and bone was set, and there was no frictional contact between the broken ends of the fracture. A force of Fx $=300 \mathrm{~N}, \mathrm{Fz}=-600 \mathrm{~N}$ was applied to the top of the femoral head, and the distal femur was fixed with 6 degrees of freedom set to 0 . The evaluation criteria included femoral displacement and the maximum von Mises (equivalent) stress of the OBS.

\section{Optimal three-rod position relationship}

The arc of the third rod was assembled near the great trochanter. Then, starting from the lateral recess of the femur, the mechanical properties of the third rod moving down $0 \mathrm{~mm}, 10 \mathrm{~mm}, 20 \mathrm{~mm}, 25 \mathrm{~mm}, 30 \mathrm{~mm}, 35 \mathrm{~mm}$, and $50 \mathrm{~mm}$ were analyzed, respectively, and the optimal position of the third rod was analyzed (see Figure 3).

\section{Optimal cross angle of proximal screw}

According to the optimal position of the third rod and the flexibility of the proximal connecting block $\left(^{\circ}\right)$ of the third rod, the direction of the proximal screw was adjusted accordingly. According to the position in Fig. 4, the optimal intersection angle of the proximal screw was analyzed by gradient analysis at a spatial angle of $3 \circ$.

\section{Optimal diameter of connecting rod}

The OBS connecting rod has two specifications: $5 \mathrm{~mm}$ and $6 \mathrm{~mm}$. Two fixing models of connecting rods are used respectively to analyze the optimal diameter of connecting rod.

\section{Optimal screw number scheme}

According to the optimal position of the third rod and the optimal intersection angle of proximal screws analyzed in the previous two steps, the fixation model with the different number of screws was used to analyze the optimal number of screws. (Fig. 5)

\section{Results}

\section{Verification of scheme validity}

The same bone condition was used to simulate a femoral shaft fracture at the same site, and the fixation was performed with a plate (see Figure 6). The comparison results with the OBS are shown in Table 1. In the OBS treatment of PPFs, the stress at the fracture end was lower than that of simple femoral shaft fractures fixed with steel plate, which proved that the fixation was effective.

\section{Optimal third rod position}

The analysis results were shown in Table 2. With the downward movement of the third rod, the femoral displace- ment generally showed a decreasing trend, while the maximum von Mises stress of the OBS showed an increasing trend. Thus, the comprehensive analysis showed that the femoral displacement and the maximum von Mises stress of the OBS were the minimum at the downward movement of the third rod $35 \mathrm{~mm}$.

\section{Optimal cross angle of proximal screw}

The analysis results were shown in Figure 7. When the spatial angle between the proximal third rod screw and the first and second-rod fixation screws was 71.92。, the femoral displacement and the maximum von Mises stress of OBS were the minima in the comprehensive analysis. 
Optimal connection rod diameter

The results are shown in Table 3. Femoral displacement and the maximum von Mises stress of OBS are smaller when using a $6 \mathrm{~mm}$ diameter connecting rod than when using a $5 \mathrm{~mm}$ connecting rod.

\section{Optimum screws number scheme}

The results were shown in Table 4. Again, scheme F had the most significant number of screws, and both femoral displacement and the maximum von Mises stress of OBS were the smallest.

\section{Discussion}

There are various methods of internal fixation for fractures around the Vancouver type B1 prosthesis. Still, due to the presence of the intramedullary prosthesis, the double cortical fixation cannot be achieved, the holding power of the internal fixation is reduced, and the con to reoperation 19,23 . The unique locking structure of OBS consists of a pin, rod, and block, which are freely matched to form a plurality of internal fixation complexes. Different from the single position and direction of plate series screws, the position and direction of OBS screws can be adjusted and controlled at will according to the situation, providing wider operability and applicability for the treatment of PPFs-thus making up for the shortcomings of other internal fixation devices. The multi-rod fixation mode and cross-screw fixation of OBS can achieve three- dimensional fixation and further improve the fixation strength. The rod-block combination can better disperse stress, avoid stress concentration and reduce the fracture risk of the internal fixator24. In addition, intraoperative soft tissue dissection is too much; the blood supply is reduced and can easily heal the fracture. Therefore, the application of the OBS can be achieved through a minimally invasive or limited incision operation, with minimal damage to soft tissue and periosteum. Bridging fixation does not directly compress the periosteum and fracture site, which has little impact on the blood supply of the fracture area and protects the biological environment of the fracture area25. An animal experiment also showed that OBS could effectively reduce the disruption of blood supply at the fracture site and provide a firm fixation26. The OBS connection block can slide axially to achieve compression and has the function of a "reset device," which can be fixed by changing the reduction edge during surgical operation 27.

The use of OBS in the treatment of Vancouver type B1 periprosthetic fracture of the femur has proven to be solid and reliable with satisfactory clinical results 28. Due to the flexibility of the OBS combination mode, this experiment was designed to explore the optimal combination mode. The OBS rodblock structure allows the screw position to be adjusted freely according to the specific situation, and the pressure hole or lock hole can be selected freely. The angular screws have better pull-out resistance and fixed strength15. The three-dimensional fixation of the OBS can be achieved when the three-rod fixation is used. The third rod is about $20 \mathrm{~mm}$ below the lateral concave of the femur, and the femoral displacement and the maximum von Mises stress of the OBS are both set to the maximum. The failure possibility of internal fixation is the highest when the third rod is placed there. The lower $35 \mathrm{~mm}$ femoral deformation and the OBS stress are minimal, the best third-rod placement position. The intersection angle of the proximal screw on the femoral displacement and the maximum von Mises stress of the OBS is generally perfect. Still, there are two trough points; the intersection angle is about $71 \circ$ and $84 \circ$, respectively. We believe that both of these two cases are feasible. To fix the strength, choosing a $6 \mathrm{~mm}$ connecting rod with low femoral displacement and the maximum von Mises stress of OBS is recommended. Although the number of screws was the most stable, considering the complexity of the clinical operation, we believed that the plan D with little difference was the best plan for the number of screws.

This study has its limitations. The study included subjects whose done mass did not accurately reflect the bone mass in patients with PPFs. In addition, the proximal femur bone mineral density will decrease due to the stress shielding effect, and the Gruen7 area has the greatest chance of decrease29,30. However, bone loss was not considered in this experiment. Muscle and other soft tissues were not considered in this study, and only biomechanical evaluation under the same bone and the same load was performed.

\section{Conclusion}

The personalized and diversified fixation mode of the OBS is well adapted to the characteristics of PFF and provides an effective means for the treatment of the periprosthetic femoral fracture.

\section{Declarations}

Ethical Approved : Approved by Yan'an Hospital Affiliated to Kunming Medical University Ethical Commit- tee, Kunming, Yunnan, China.

Declarations: Not applicable

Funding: This work was supported by Kunming Health Committee [2019-04-07-007] [4] and Yunnan Health Committee [H-2019014], Yunnan, China.

Conflicts of interest/Competing interests: The authors declare that they have no competing interests.

Data availability: The data sets used and analyzed during the current study are available from the correspond-ing author on reasonable request.

Author contributions: LZ, MAH \& JX, Substantial contributions to conceptionand design. All authors: Data acquisition, data analysis and interpretation. All authors: Drafting the article or critically revising it for important intellectual content. All authors: Final approval of the version to be published. JQ, LL,YZ and YX: Agreement to be accountable for all aspects of the work in ensuring that questions related to the accuracy or integrity of the work are appropriately investigated and resolved. 
Statement: We confirm that this manuscript has not been published or presented elsewhere in part or entirety and is not under consideration by another journal. However you noticed that a preprint has been published in research square https://www .researchsquare.com/article/rs-661745/v1, "Finite element analysis of the optimal configuration of brdging combined internal fixation system in the treatment of Vancouver B1 periprosthetic femoral fractures" but its not published or under consideration by any journal.

\section{References}

[1] Steven Kurtz. "Projections of primary and revision hip and knee arthroplasty in the United States from 2005 to 2030". Jbjs, vol. 89, 780-785, 2007.

[2] Frank A Liporace. "Periprosthetic Fractures About the Hip and Knee: Contemporary Techniques for Internal Fixation and Revision". Instructional course lectures, vol. 67, 223-239, 2018.

[3] M P Abdel. "Epidemiology of periprosthetic fracture of the femur in 32644 primary total hip arthroplasties: a 40-year experience". The bone \& joint journal, vol. 98, 461-467, 2016.

[4] Robert Pivec. "Incidence and future projections of periprosthetic femoral fracture following primary total hip arthroplasty: an analysis of international registry data". Journal of long-term effects of medical implants, vol. 25, 2015.

[5] A Aprato. "The clinical and economic burden of proximal femur periprosthetic fractures". Musculoskeletal surgery, pages 1-6, 2021.

[6] G Agostini. "Clinical and radiographical outcome after surgical treatment of periprosthetic type B proximal femur fractures: a retrospective study". Musculoskeletal surgery, pages 1-5, 2020.

[7] Owen H Brady. "The reliability of validity of the Vancouver classification of femoral fractures after hip replacement". The Journal of arthroplasty, vol. 15, $59-62,2000$.

[8] Jeffrey Pike. "Principles of treatment for periprosthetic femoral shaft fractures around well-fixed total hip arthroplasty". JAAOS-Journal of the American Academy of Orthopaedic Surgeons, vol. 17, 677-688, 2009.

[9] Jagannath Chakravarthy, Rajeev Bansal and Julian Cooper. "Locking plate osteosynthesis for Vancouver Type B1 and Type C periprosthetic fractures of femur: a report on 12 patients". Injury, vol. 38, 725-733, 2007.

[10] Matthew P Abdel, Umberto Cottino and Tad M Mabry. "Management of periprosthetic femoral fractures following total hip arthroplasty: a review". International orthopaedics, vol. 39, 2005-2010, 2015.

[11] Tanvir Khan. "High mortality following revision hip arthroplasty for periprosthetic femoral fracture: a cohort study using National Joint Registry data". The Bone \& Joint Journal, vol. 102, 1670-1674, 2020.

[12] B F El-Zayat. "NCB-plating in the treatment of geriatric and periprosthetic femoral fractures". Orthopaedics \& Traumatology: Surgery \& Research, vol. 98, $765-772,2012$.

[13] Sanjeev Agarwal, C M Andrews and G M Bakeer. "Outcome following stabilization of type B1 periprosthetic femoral fractures". The Journal of arthroplasty, vol. 20, 118-121, 2005.

[14] Theerachai Apivatthakakul. "Percutaneous cerclage wiring and minimally invasive plate osteosynthesis (MIPO): a percutaneous reduction technique in the treatment of Vancouver type B1 periprosthetic femoral shaft fractures". Archives of orthopaedic and trauma surgery, vol. 132, 813-822, 2012.

[15] Martin F Hoffmann. "Biomechanical evaluation of fracture fixation constructs using a variable-angle locked periprosthetic femur plate system". Injury, vol. 45, 1035-1041, 2014.

[16] E Gautier. "Sommer Guidelines for the clinical application of the LCP. C". Injury, vol. 34, no. 2, 2003.

[17] M A Buttaro. "Locking compression plate fixation of Vancouver type-B1 periprosthetic femoral fractures".

JBJS, vol. 89, 1964-1969, 2007.

[18] Fares S Haddad. "Periprosthetic femoral fractures around well-fixed implants: use of cortical onlay allografts with or without a plate". JBJS, vol. 84, 945-950, 2002.

[19] Wood and C A Gavin. "Locking compression plates for the treatment of periprosthetic femoral fractures around well-fixed total hip and knee implants". The Journal of arthroplasty, vol. 26, 886-892, 2011.

[20] Qi Chen. "Treatment of Vancouver type B1 periprosthetic femoral fracture with locked plate and xenogenic bony plate". Zhongguo gu shang= China journal of orthopaedics and traumatology, vol. 29, 734-737, 2016. 
[21] Karl Stoffel. "The influence of the operation technique and implant used in the treatment of periprosthetic hip and interprosthetic femur fractures: a systematic literature review of 1571 cases". Archives of orthopaedic and trauma surgery, vol. 136, 553-561, 2016.

[22] S G Yan. "Finite element analysis of femoral stress changes before and after total hip arthroplasty". Zhonghua Gu Shang, pages 52-56, 2004.

[23] Neil P Sheth. "Operative treatment of early peri-prosthetic femur fractures following primary total hip arthro- plasty". The Journal of arthroplasty, vol. 28, 286-291, 2013.

[24] Wang and Da-Xing. "Biomechanical analysis and clinical effects of bridge combined fixation system for femoral fractures". Proceedings of the Institution of Mechanical Engineers, vol. 228, 899-907, 2014.

[25] Liangqi Kang. "Ipsilateral proximal and shaft femoral fractures treated with bridge-link type combined fixa- tion system". Journal of Orthopaedic Surgery and Research, vol. 15, 1-10, 2020.

[26] Ying Xiong. "Effects of bridge combined fixation system on healing of fractures in dogs". Journal of Clinical Rehabilitative Tissue Engineering Research, vol. 15, 5556-5560, 2011.

[27] Zhi-Qiang Lv, Xing-Hua Li and Ai-Guo Wang. "Repair of femoral shaft fracture with bridging combined internal fixation and locking plate screw system: a biomechanical comparison”. Chinese Journal of Tissue Engineering Research, vol. 20, 2555-2555, 2016.

[28] M Z Wang. "The bridging combined internal fixation system for the treatment of Vancouver type B1 peripros- thetic femoral fractures". Chinese Journal of Traumatic Orthopedics, vol. 22, 804-807, 2020.

[29] Ashleen R Knutsen. "Periprosthetic femoral bone loss in total hip arthroplasty: systematic analysis of the effect of stem design". Hip International, vol. $27,26-34,2017$.

[30] Toni Tapaninen, Heikki Kroger and Petri Venesmaa. "Periprosthetic BMD after cemented uncemented total hip arthroplasty: a 10-year follow-up study". Journal of Orthopaedic Science, vol. 20, 657-662, 2015.

\section{Tables}

Due to technical limitations, the tables can be found as a download in the supplementary files section.

\section{Figures}

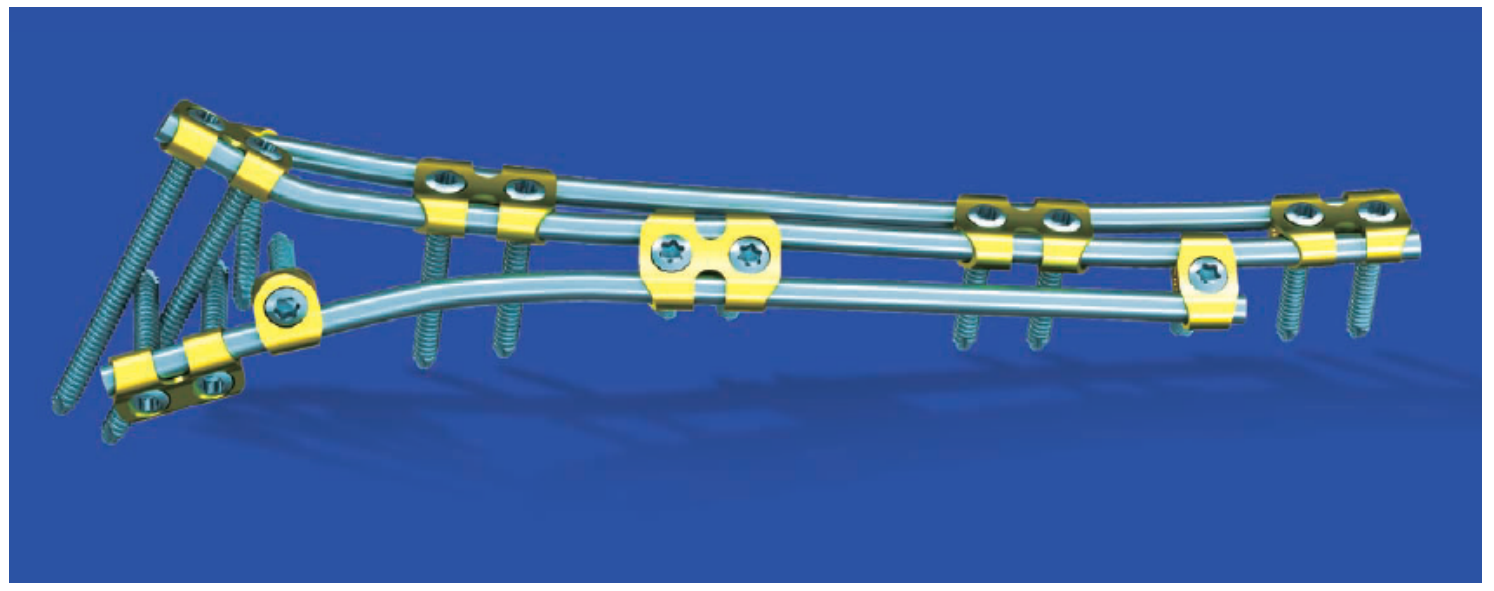

Figure 1

Bridged combined internal fixation system (Ortho-bridge System, OBS) 


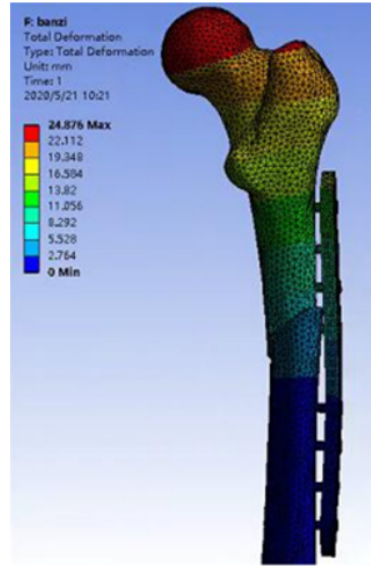

(a)

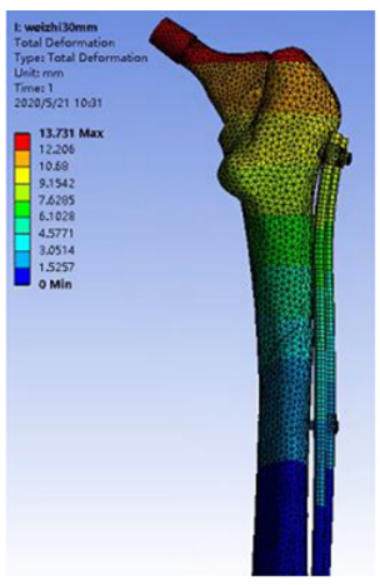

(c)

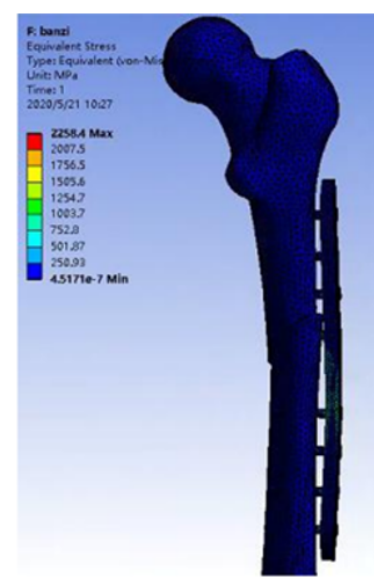

(b)

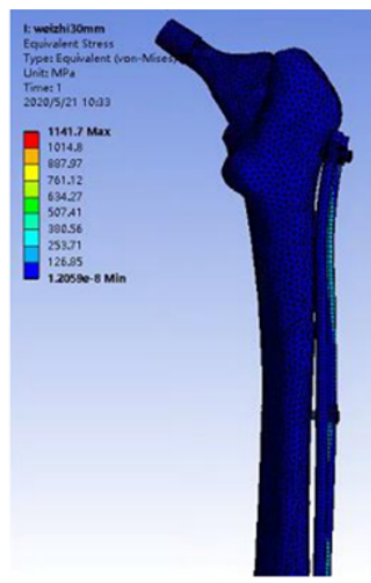

(d)

\section{Figure 2}

Verification of scheme validity(a: femoral displacement fixed by steel plate, b: stress distribution diagram for steel plate fixation, c: femoral displacement fixed by OBS, d: Stress distribution diagram for OBS fixation)

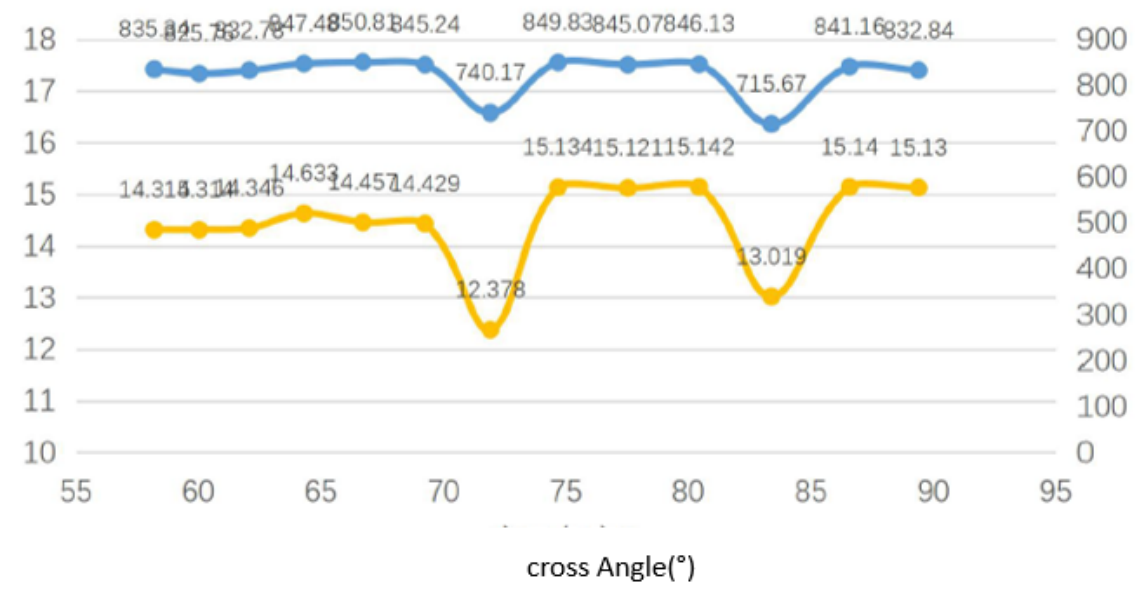

$\rightarrow$ the maximum Von Mises stress of OBS(MPa) - femoral displacement $(\mathrm{mm})$

\section{Figure 3}

Relationship between femoral displacement, the maximum Von Mises stress of OBS and cross Angle of proximal screw 


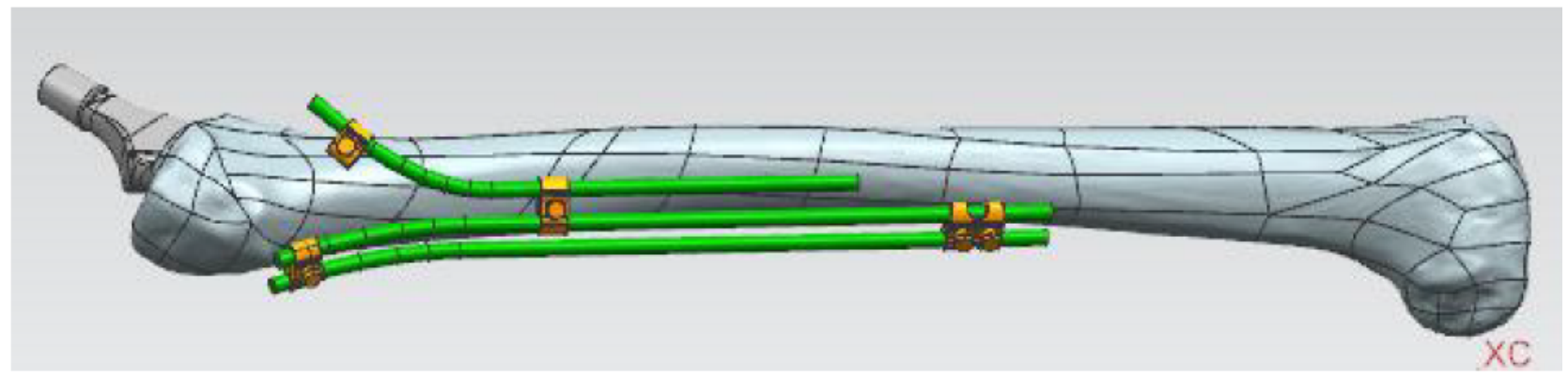

\section{Figure 4}

Periprosthetic femoral fracture model with OBS fixation

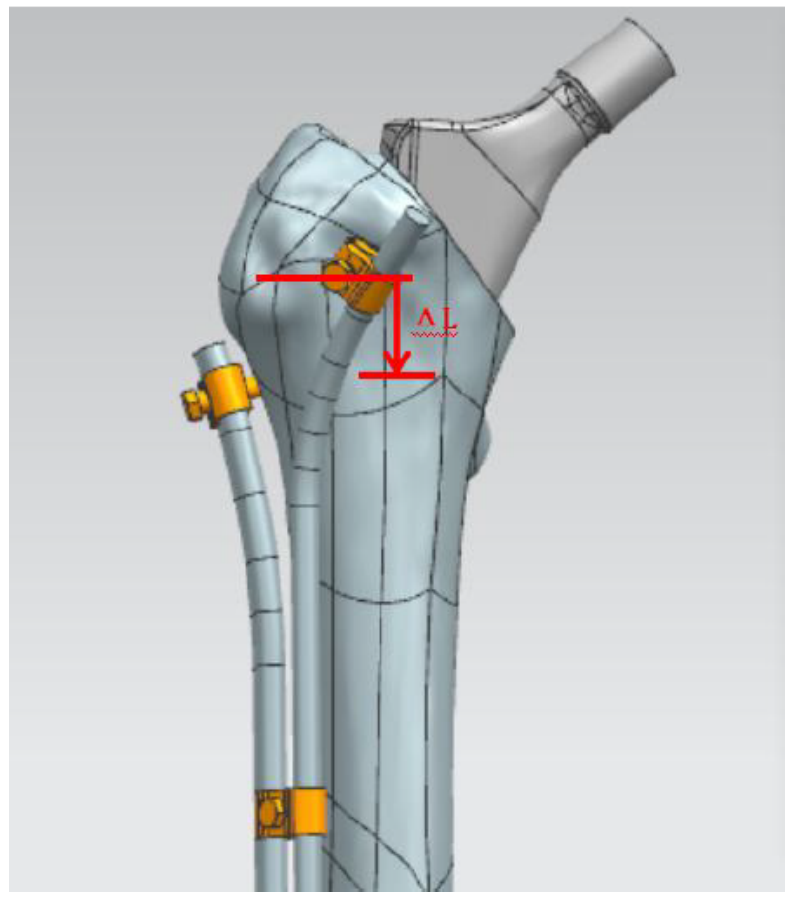

\section{Figure 5}

Position design of the third rod

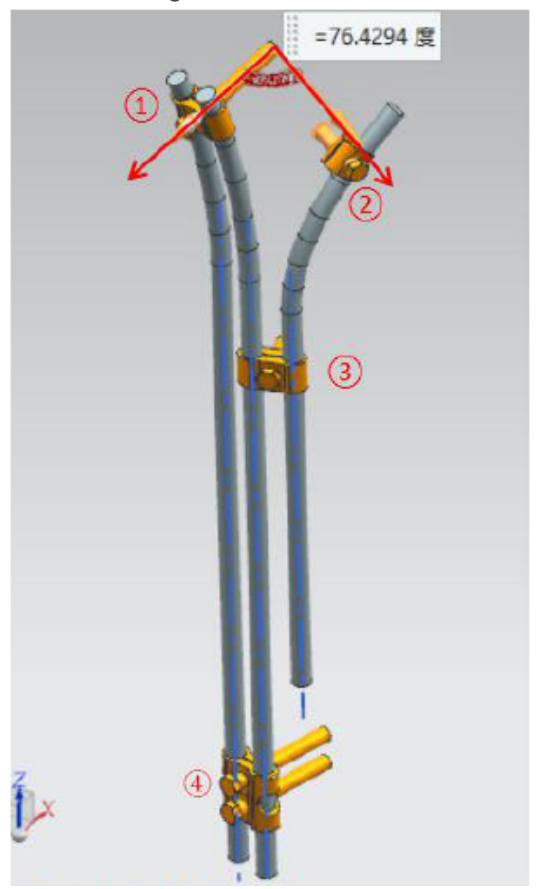


Figure 6

Cross Angle of proximal screw

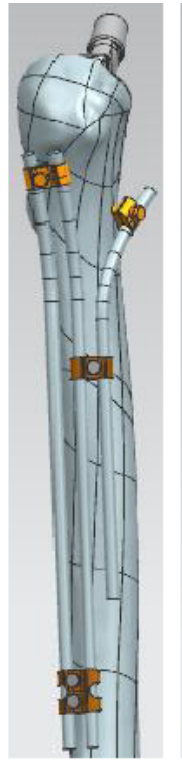

(a)

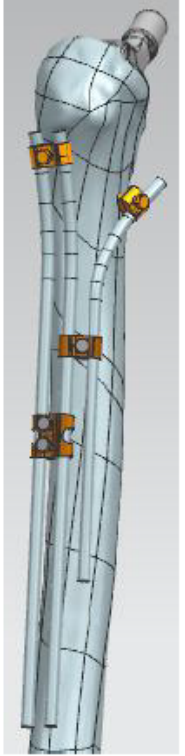

(b)

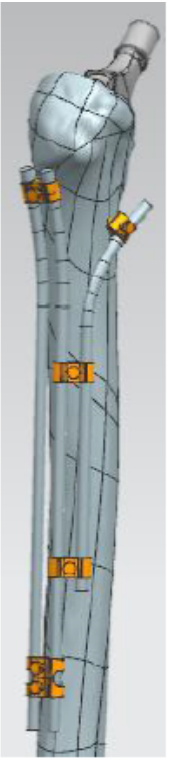

(c)

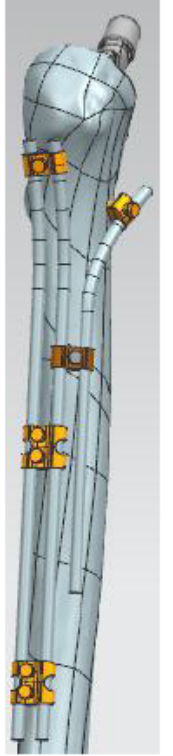

(d)

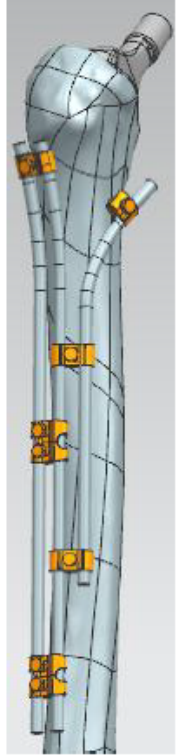

(e)

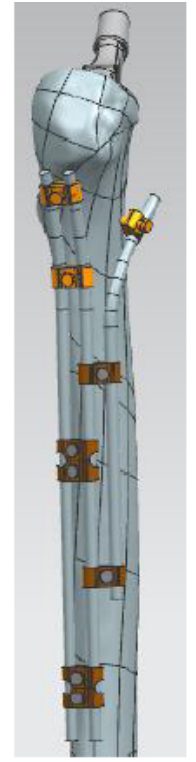

(f)

Figure 7

screw number scheme

\section{Supplementary Files}

This is a list of supplementary files associated with this preprint. Click to download.

- tables.docx 